\title{
Del pasmo al marasmo : El sector de la construcción y su relación con la crisis del empleo
}

\section{CARLOS GARCÍA SERRANO}

Departamento de Fundamentos de Economía e Historia Económica, UNIVERSIDAD DE ALCALÁ, ESPAÑA. E-mail: carlos.garcia@uah.es

\begin{abstract}
RESUMEN
Este artículo examina la evolución del sector de la construcción desde la perspectiva de la ocupación. Para ello, se adoptan dos puntos de vista relevantes: uno de ellos es el temporal, lo que lleva a analizar cómo ha evolucionado la estructura sectorial, en general, y el nivel de empleo de la construcción y ramas afines, en particular, en España en las últimas décadas; la otra perspectiva es la internacional, lo que implica comparar a España con otros países, sobre todo europeos, y preguntarse si el caso español ha sido muy diferente al del resto.
\end{abstract}

Palabras clave: Construcción, empleo, ciclo económico.

\section{The Construction Sector and its Relationship With the Employment Crisis}

\begin{abstract}
This article examines the evolution of the construction sector using the employment perspective. For that, it adopts two relevant points of view: one is temporal, which leads to the analysis of the evolution of the sectoral structure, in general, and of the employment level of construction and related economic activities, in particular, in Spain during the last decades; the other perspective is the international, which implies comparing Spain with other countries (especially, European countries) and inquiring whether the Spanish case has been so different from the rest.
\end{abstract}

Keywords: Construction, Employment, Business Cycle.

Clasificación JEL: J21, J40

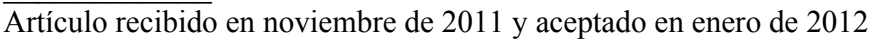

Artículo disponible en versión electrónica en la página www.revista-eea.net, ref. ə-30101 


\section{INTRODUCCIÓN}

El sector de la construcción ha sido el protagonista indiscutible del último ciclo de la economía española, del auge del empleo que tuvo lugar en el período expansivo 1995-2007 y de la caída producida en la etapa recesiva 2008-2011. Mientras algunos analistas consideran que el desmesurado desarrollo de dicho sector durante la expansión y su posterior ajuste han sido responsables fundamentales de la extraordinarias variaciones sufridas por los niveles de empleo y paro (Toharia y Malo, 2009), otros no atribuyen este papel a la construcción (Felgueroso, 2011).

Uno de los aspectos que hay que destacar sobre la evolución del sector inmobiliario y su relación con el resto de la economía en España es que los procesos de auge de dicho sector se han manifestado asociados a crecimientos más significativos de la economía y acompañados de aumentos intensos de la actividad, de los precios inmobiliarios y del empleo en el sector de la construcción, aunque es evidente que este rasgo no es distintivo de España sino que se encuentra documentado para una gran variedad de países (Englund y Ioannides, 1997; Kasparova y White, 2001). Este vínculo entre la dinámica de la actividad y los precios en la construcción y los indicadores macroeconómicos del crecimiento económico general se ha observado con claridad en las dos últimas etapas expansivas (1986-1991 y 1996-2007).

La primera estuvo vinculada a cambios introducidos en el funcionamiento de los sistemas financieros en muchos países (occidentales), al eliminarse gran cantidad de controles hasta entonces vigentes y lograrse una integración plena de los mercados hipotecarios en dichos sistemas, además de liberalizar las prácticas prestatarias (Meen, 1990, 1996; Kim, 1999). En el caso español, las reformas introducidas en el normativa del mercado hipotecario en 1981 y 1982 facilitaron la refinanciación de los préstamos hipotecarios (se desarrollaron las cédulas y los bonos hipotecarios) y acompañaron a la eliminación de las restricciones cuantitativas al crecimiento de los agregados monetarios y de los controles de los tipos de interés, cambios que permitieron el alargamiento de los plazos de los préstamos hipotecarios y un aumento de la relación préstamo/valor. Además, el Real Decreto 2/1985 (Decreto Boyer) introdujo medias estimulantes de la demanda inmobiliaria, entre las que destacó la flexibilidad total en la fijación de los alquileres. También se introdujo la desgravación fiscal a la compra de segundas viviendas y la posibilidad de transformar las viviendas en locales de negocios. El resultado de todos estos cambios fueron aumentos nominales en los precios de la vivienda del 16,3\% de promedio en 1986-1991 y de casi el $10 \%$ en términos reales, así como un crecimiento muy rápido de las viviendas iniciadas hasta 1989, y eso a pesar de que los tipos de interés permanecieron elevados durante todo el período (Rodríguez, 2006). 
En la segunda etapa, además de factores macroeconómicos (una expansión económica prolongada) y demográficos (cambios en la composición de la población y, en algunos países, aumento de la misma por efecto de los flujos migratorios), el auge ha sido sobre todo consecuencia de la perturbación de demanda que han supuesto las excepcionales condiciones de financiación a largo plazo al sector inmobiliario (Stevenson y Young, 2006). Los tipos de interés nominales descendieron a partir de la segunda parte de la década de los años noventa del siglo XX y permanecieron en general bajos como expresión de una política monetaria laxa. Dado que la inflación también se mantuvo en niveles bajos, el resultado fue la existencia de unos tipos de interés reales también muy reducidos, lo que permitió a los hogares la financiación de hipotecas mayores. A esto se añadió una mejora sustancial de las restantes condiciones de financiación por las entidades de crédito (plazo de los préstamos, relación préstamo/valor) que reforzaron la demanda de consumo y de inversión en vivienda y que permitieron el desarrollo de unas expectativas de revalorización de los inmuebles por parte de los compradores que provocaron una "burbuja" (GarcíaMontalvo, 2006, 2009). El resultado en el caso español fue un aumento excepcional tanto del número de viviendas iniciadas como la de superficie construida (por ejemplo, el aumento del parque de viviendas en 2001-2004 superó el 8\% se pasó de 20,9 millones a 22,6-) así como un incremento real de los precios cercano al 9\% anual en 1998-2006. El proceso de rápida elevación de los precios de las viviendas, de prolongada duración de dichos aumentos y de desarrollo de importantes volúmenes de construcción residencial no ha sido una exclusiva de España (Whitehead, 2006), aunque estas variables han presentado en general una mayor intensidad aquí que en el promedio de la eurozona (pero no que en Irlanda, por ejemplo) o Estados Unidos de América (FMI, 2004; OCDE, 2005; The Economist, 2005).

Más allá de los cambios en los precios o en el volumen de viviendas, el objetivo de este artículo consiste en analizar el sector de la construcción desde la perspectiva de la ocupación. Para ello, se adoptan dos puntos de vista relevantes: uno de ellos es el temporal, lo que lleva a examinar cómo ha evolucionado la estructura sectorial, en general, y el nivel de empleo de la construcción y ramas afines, en particular, en España en las últimas décadas; la otra perspectiva es la internacional, lo que implica comparar a España con otros países, sobre todo europeos, y preguntarse si el caso español ha sido muy diferente al del resto.

La estructura del artículo es la siguiente. En la sección dos, se ofrece una panorámica de la evolución sectorial del empleo en España en los últimos veinticinco años, con especial énfasis en los cambios acaecidos en el sector de la construcción. En la sección tres, se presenta una descripción de la evolución de la ocupación en España en comparación con el resto de los países de la Unión 
Europea para tratar de detectar algún rasgo distintivo de aquélla en comparación con éstos. Finalmente, la sección cuatro ofrece las conclusiones.

\section{LA EVOLUCIÓN SECTORIAL DEL EMPLEO EN ESPAÑA}

Durante los últimos veinticinco años, España ha vivido dos crisis del empleo de gran magnitud (1991-1994 y 2008-2011) y dos etapas de expansión muy intensa (1985-1991 y 1995-2007). Estos vaivenes han dejado su huella en la composición sectorial del empleo, como puede comprobarse con la información proporcionada en la Tabla 1 y en la Figura 1, a partir de los datos publicados de la Encuesta de Población Activa (EPA) ${ }^{1}$. La Tabla 1 ofrece la distribución de los ocupados en siete grandes grupos de actividad económica en los momentos que pueden considerarse los puntos de inflexión del ciclo a lo largo de este período (1985, 1991, 1994, 2007 y 2011), así como la variación absoluta y la variación relativa del empleo en esos sectores durante el período 1985-2011 dividido en las diferentes etapas de expansión y recesión. Los sectores considerados son los siguientes: "agricultura", "industria", "construcción", "servicios tradicionales" (comercio, hostelería, transportes y comunicaciones), "servicios avanzados" (actividades financieras, actividades inmobiliarias y servicios a las empresas), "servicios colectivos" (administración pública, educación y sanidad) y "otros servicios" (actividades artísticas, otros servicios personales, hogares que emplean personal doméstico y organismos extraterritoriales). Además, la Figura 1 complementa la información anterior ofreciendo la evolución del empleo total y del empleo en la construcción así como el peso de este sector con respecto a la ocupación total y con respecto a la ocupación no agraria a lo largo de todo el período.

Los datos de la Tabla permiten obtener una visión de largo plazo del cambio sectorial operado en la economía española desde mitad de los años ochenta del siglo XX. De ellos se deduce que la reasignación sectorial del factor trabajo se ha centrado fundamentalmente en los siguientes aspectos.

En primer lugar, una caída continua del empleo agrario en términos absolutos ( 1,2 millones menos de ocupados, más del $60 \%$ de la ocupación que tenía el sector en 1985), lo que ha llevado a que su peso haya disminuido de más del 18\% del empleo total en 1985 al 4\% en 2011.

\footnotetext{
${ }^{1}$ Debe tenerse en cuenta que a lo largo del período considerado se han producido dos cambios en la clasificación de las ramas de actividad: a partir del primer trimestre de 1993 comenzó a emplearse la Clasificación Nacional de Actividades Económicas 1993 (CNAE-1993) y a partir del primer trimestre de 2009 la nueva Clasificación Nacional de Actividades Económicas 2009 (CNAE-2009). En la medida en que aquí se utilizan agregaciones suficientemente amplias, cabe suponer que estos cambios no han tenido efectos importantes en el cómputo de los niveles de empleo y en sus variaciones para los sectores considerados.
} 
En segundo lugar, una reducción de la importancia del empleo industrial debido a que durante las recesiones este sector pierde empleo mientras otros lo ganan, lo que hace disminuir su peso, y durante las expansiones aumenta su empleo pero a un ritmo menor que otros sectores y que el total de la economía, por lo que también se reduce su peso. En conjunto, la ocupación en la industria es la misma en 2011 que en 1985 y su participación en el empleo total ha caído desde casi el $25 \%$ en 1985 al $14 \%$ en 2011 , caída concentrada especialmente en el período expansivo 1995-2007. Esta disminución se revela más notable cuando se considera la distribución del empleo no agrario en vez del empleo total.

En tercer lugar, un aumento del empleo en los servicios, con experiencias algo diferentes dependiendo del sector considerado. Los dos sectores que más han incrementado su empleo neto en términos absolutos en el conjunto del período han sido los servicios colectivos y los servicios tradicionales $(2,7$ y 3 millones, respectivamente), pero mientras los primeros vieron aumentar su ocupación en cada subperíodo, los segundos concentraron los incrementos del empleo en las etapas expansivas reduciéndolo muy ligeramente en las recesivas. En cualquier caso, el peso de ambos en el empleo total ha aumentado de forma relevante, desde el 13,3\% en 1985 al 22,2\% en 2011 en el caso de los servicios colectivos y desde el $25,7 \%$ al $31,5 \%$ en el caso de los servicios tradicionales, si bien la variación es más matizada en este último caso cuando se considera la distribución del empleo no agrario. El sector de otros servicios se ha comportado de forma similar al de los servicios tradicionales, con aumentos de la ocupación en las expansiones (particularmente en 1995-2007) y reducciones en las recesiones.

Tabla 1

Distribución del empleo en España por ramas de actividad y variación absoluta (en miles de personas) y relativa, 1985-2011

\begin{tabular}{|l|r|r|r|r|r|r|r|r|r|r|}
\hline \multicolumn{1}{|c|}{ DISTRIBUCIÓN } & \multicolumn{4}{c|}{ Empleo total } & \multicolumn{4}{c|}{ Empleo no agrario } \\
\hline & $\mathbf{1 9 8 5}$ & $\mathbf{1 9 9 1}$ & $\mathbf{1 9 9 4}$ & $\mathbf{2 0 0 7}$ & $\mathbf{2 0 1 1}$ & $\mathbf{1 9 8 5}$ & $\mathbf{1 9 9 1}$ & $\mathbf{1 9 9 4}$ & $\mathbf{2 0 0 7}$ & $\mathbf{2 0 1 1}$ \\
\hline Agricultura & 18,4 & 10,5 & 9,5 & $\mathbf{4 , 5}$ & $\mathbf{4 , 0}$ & - & - & - & - & - \\
\hline Industria & 24,5 & 23,0 & 21,1 & 15,9 & 14,1 & 30,0 & 25,7 & 23,3 & 16,7 & 14,7 \\
\hline Construcción & 7,3 & 10,3 & 9,2 & 13,3 & 7,8 & 8,9 & 11,5 & 10,1 & 14,0 & 8,1 \\
\hline Servicios tradicionales & 25,7 & 27,9 & 29,1 & 28,3 & 31,5 & 31,5 & 31,2 & 32,2 & 29,6 & 32,8 \\
\hline Servicios avanzados & 4,2 & 7,1 & 7,8 & 12,3 & 12,6 & 5,2 & 8,0 & 8,6 & 12,9 & 13,1 \\
\hline Servicios colectivos & 13,3 & 15,1 & 17,0 & 17,7 & 22,2 & 16,3 & 16,9 & 18,8 & 18,5 & 23,1 \\
\hline Otros servicios & 6,6 & 6,1 & 6,3 & 8,0 & 7,8 & 8,0 & 6,8 & 6,9 & 8,4 & 8,2 \\
\hline Total & 100,0 & 100,0 & 100,0 & 100,0 & 100,0 & 100,0 & 100,0 & 100,0 & 100,0 & 100,0 \\
\hline Empleo (miles) & 10.583 & 13.068 & 12.186 & 20.367 & 18.303 & 8.638 & 11.694 & 11.030 & 19.446 & 17.562 \\
\hline
\end{tabular}


Tabla 1 (continuación)

Distribución del empleo en España por ramas de actividad y variación absoluta (en miles de personas) y relativa, 1985-2011

\begin{tabular}{|l|r|r|r|r|r|r|r|r|r|r|}
\hline VARIACIÓN & \multicolumn{2}{|c|}{$1985-1991$} & \multicolumn{2}{|c|}{$1991-1994$} & \multicolumn{2}{|c|}{$1994-2007$} & \multicolumn{2}{|c|}{$\mathbf{2 0 0 7 - 2 0 1 1}$} & \multicolumn{2}{c|}{ Total } \\
\hline & Var.abs & \%Var. & Var.abs & \%Var. & Var.abs & \%Var. & Var.abs & \%Var. & Var.abs & $\%$ Var. \\
\hline Agricultura & -571 & $-29,3$ & -217 & $-15,8$ & -236 & $-20,4$ & -180 & $-19,5$ & -1.203 & $-61,9$ \\
\hline Industria & 411 & 15,9 & -434 & $-14,5$ & 676 & 26,3 & -666 & $-20,5$ & -13 & $-0,5$ \\
\hline Construcción & 571 & 74,0 & -224 & $-16,7$ & 1.595 & 142,5 & -1.284 & $-47,3$ & 658 & 85,3 \\
\hline Servicios tradicionales & 923 & 33,9 & -96 & $-2,6$ & 2.212 & 62,3 & -1 & 0,0 & 3.037 & 111,5 \\
\hline Servicios avanzados & 486 & 108,7 & 21 & 2,2 & 1.549 & 162,6 & -200 & $-8,0$ & 1.856 & 415,4 \\
\hline Servicios colectivos & 565 & 40,1 & 101 & 5,1 & 1.522 & 73,3 & 457 & 12,7 & 2.645 & 187,6 \\
\hline Otros servicios & 100 & 14,5 & -32 & $-4,0$ & 863 & 113,3 & -191 & $-11,7$ & 741 & 106,8 \\
\hline Total & 2.485 & 23,5 & -882 & $-6,7$ & 8.181 & 67,1 & -2.064 & $-10,1$ & 7.721 & 73,0 \\
\hline
\end{tabular}

Nota: "servicios tradicionales" incluye comercio, reparaciones, hosteleria, transportes y comunicaciones; "servicios avanzados" incluye intermediación financiera, actividades inmobiliarias y de alquiler y servicios a empresas; "servicios colectivos" incluye administración pública, educación, actividades sanitarias y organismos extraterritoriales; "otros servicios" incluye otras actividades sociales y hogares que emplean personal doméstico.

Fuente: EPA, $2^{\circ}$ trimestres (INE).

Finalmente, mención especial merece la evolución de los servicios avanzados, que en conjunto aumentaron su empleo neto en más de 1,8 millones de personas, con incrementos durante todas las subetapas (pero sobre todo en 1995-2007) excepto durante la recesión actual (con una caída del 8\% del empleo que tenían en 2007). Aun así, el peso de este sector ha pasado de un $4,2 \%$ del empleo total en 1985 a un $12,6 \%$ en 2011. Este sector es relevante para el tema que nos ocupa puesto que incluye las ramas que tienen que ver con las actividades financieras, las actividades inmobiliarias, el alquiler de maquinaria y equipo y los servicios a las empresas, actividades que en parte se encuentran vinculadas directa o indirectamente con el sector de la construcción. $\mathrm{El}$ auge de este sector se ha concentrado especialmente en el subperíodo expansivo 1995-2007 (prácticamente todo el aumento del empleo neto y la mayor parte del aumento de la participación del sector en la ocupación total se producen en dicho subperíodo) en el que, como ahora veremos, también se ha producido el mayor incremento del sector de la construcción. De hecho, cuando se considera de forma desagregada el subsector de actividades inmobiliarias (rama 70 de la CNAE), su importancia en el empleo total se mantuvo en el $0,2 \%-0,3 \%$ desde 1985 hasta 1995 , en torno al $0,4 \%$ en la segunda mitad de los noventa y luego fue aumentando progresivamente hasta alcanzar el 0,9\%-1,0\% en 2006-2008 para caer nuevamente al 0,5\% en 2009-2010.

Con respecto a la construcción, de la información mostrada tanto en la Tabla como en la Figura se deduce la evolución marcadamente cíclica del empleo en el sector, con un aumento importante de su ocupación y de su peso durante las 
expansiones y una reducción igualmente relevante durante las recesiones: sus variaciones relativas (positivas y negativas) son siempre mayores que las del total de la economía y que la mayoría del resto de los sectores ${ }^{2}$. En particular, desde 1985 hasta 1991 la construcción creó casi 600 mil empleos netos y su peso pasó del 7,3\% al 10,3\% del empleo total, aumentando su ocupación a un ritmo más rápido que el total de la economía, después de haber perdido empleo de forma ininterrumpida desde 1977 (véase Toharia et al., 1991). Con la llegada de la crisis económica de principios de los noventa, el sector perdió más de 200 mil efectivos y su peso retrocedió al 9,2\% del empleo total. Posteriormente, la expansión económica llevó a la construcción a ganar 1,6 millones de ocupados, aumentando su importancia en el empleo total hasta el 13,3\% en 2007. Finalmente, la recesión actual ha llevado al sector a perder casi 1,3 millones de puestos, lo que ha provocado una reducción de su peso hasta situarse en el 7,8\%, es decir, muy cerca del peso que tenía en 1985 .

Figura 1

Evolución del empleo total y del empleo en la construcción (en miles de personas) y del peso de este sector con respecto a la ocupación total y con respecto a la ocupación no agraria, 1985-2011

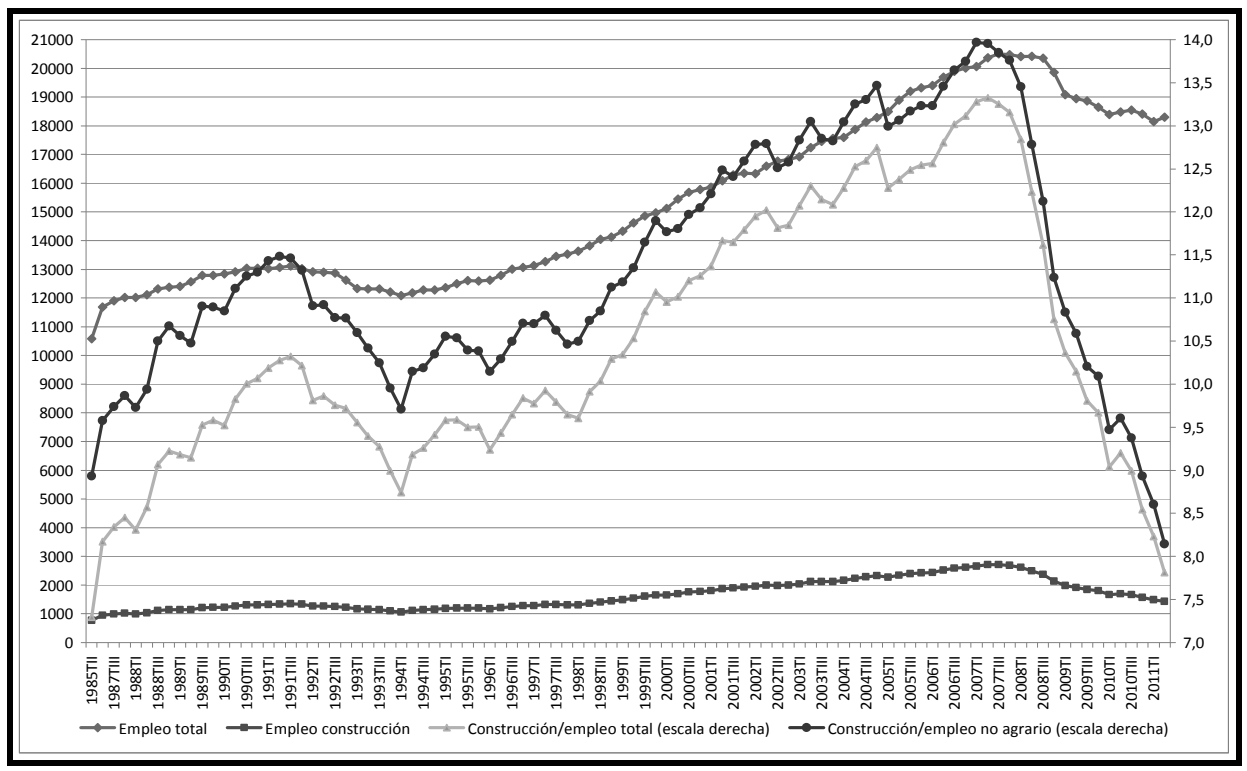

Fuente: EPA (INE).

${ }^{2}$ Esto mismo sucede con el PIB, de modo que las etapas de mayor ritmo de crecimiento de la economía española han coincidido con fases de fuerte expansión del conjunto de la construcción (véase Rodríguez, 2006). Esta característica puede estar relacionada en parte con la naturaleza de activo cíclico de los bienes inmuebles (Malpezzi y Wachter, 2005). 
La ciclicidad de la construcción es incluso más clara cuando se considera la ocupación no agraria, comparación que resulta más conveniente dado que el empleo en la agricultura ha venido reduciéndose continuamente a lo largo de todo el período. En este caso, la importancia de la construcción era el 8,96\% en 1985, alcanzó el 11,5\% en 1991, disminuyó al 10,1\% en 1994, creció hasta el $14 \%$ en 2007 y cayó al 8,1\% en 2011.

Dado este carácter cíclico de la actividad constructora, un aspecto que merece la pena explorar es si la recesión actual ha sido similar en comparación con la de principios de los noventa en cuanto a su comienzo y posterior desarrollo, es decir, si la reducción del empleo comenzó en las mismas ramas de actividad económica y posteriormente se extendió al resto. Para examinar esta cuestión en detalle, la Tabla 2 presenta el desglose sectorial de la evolución de la ocupación de las dos últimas crisis de empleo dividido en dos etapas: el comienzo (aproximadamente el primer año y medio) y el resto. En el caso de la crisis de los noventa, la etapa inicial y la etapa restante corresponden a los períodos $2^{\circ} / 1991-3^{\circ} / 1992$ y $3^{\circ} / 1992-2^{\circ} / 1994$ mientras que en el caso de la crisis actual los períodos son $2^{\circ} / 2007-3^{\circ} / 2008$ y $3^{\circ} / 2008-2^{\circ} / 2011$, respectivamente.

A la vista de los datos, cabe deducir que la crisis de principios de los noventa fue tanto en su fase inicial como en su fase posterior una crisis principalmente industrial que también afectó a la construcción, sobre todo a partir de la finalización de las obras públicas relacionadas con acontecimientos deportivosculturales en 1992, de tal forma que este sector perdió más empleo en la segunda fase que en la primera. Pero en realidad tanto en la primera fase como, sobre todo, en la segunda, la mayor parte de la reducción neta del empleo puede atribuirse al sector industrial (véase Toharia, 1996a, 1996b).

La secuencia de acontecimientos en la crisis actual es bastante diferente. Al comienzo apenas se perdieron empleos, pero mientras las pérdidas fueron relativamente pequeñas en la industria (16 mil) y en la agricultura $(72 \mathrm{mil})$, la construcción acumuló una pérdida de 350 mil (un 13\% del empleo en el sector), compensada parcialmente por los servicios con un aumento de 418 mil empleos. Por consiguiente, la crisis de empleo actual se inició con el pinchazo de la burbuja inmobiliaria y la destrucción de empleo en la construcción, concentrándose la caída de la ocupación de forma mayoritaria en este sector cuando se considera la primera fase de la crisis. En la segunda parte, la caída de la ocupación fue mucho más intensa (concentrada en el otoño-invierno de 2008-2009) y, aunque la construcción fue el sector que siguió predominando en explicarla (destruyó más de 900 mil empleos, casi un $40 \%$ de la ocupación que tenía en el tercer trimestre de 2008), otros sectores se unieron a la destrucción masiva de empleos: la industria cayó de forma intensa (pérdida de 650 mil ocupados) e incluso los servicios (sobre todo actividades financieras, actividades inmobilia- 
rias y servicios a las empresas) perdieron empleo (724 mil), si bien los servicios colectivos continuaron con la creación neta de puestos $(371 \mathrm{mil})$.

\section{Tabla 2}

Evolución del empleo en España por ramas de actividad en las dos últimas crisis, por etapas (variación absoluta en miles de personas y variación relativa)

\begin{tabular}{|l|r|r|r|r|r|r|r|r|}
\hline & \multicolumn{3}{|c|}{ Crisis 1991-1994 } & \multicolumn{3}{c|}{ Crisis 2007-2011 } \\
\hline & \multicolumn{2}{|c|}{$\begin{array}{c}\text { Variación } \\
\text { absoluta }\end{array}$} & \multicolumn{2}{c|}{$\begin{array}{c}\text { Variación } \\
\text { relativa }\end{array}$} & \multicolumn{2}{c|}{$\begin{array}{c}\text { Variación } \\
\text { absoluta }\end{array}$} & \multicolumn{3}{c|}{$\begin{array}{c}\text { Variación } \\
\text { relativa }\end{array}$} \\
\hline & $\begin{array}{r}2^{\circ}-91 / \\
3^{\circ}-92\end{array}$ & $\begin{array}{r}3^{\circ}-92 / \\
2^{\circ}-94\end{array}$ & $\begin{array}{r}2^{\circ}-91 / \\
3^{\circ}-92\end{array}$ & $\begin{array}{r}3^{\circ}-92 / \\
2^{\circ}-94\end{array}$ & $\begin{array}{r}2^{\circ}-07 / \\
3^{\circ}-08\end{array}$ & $\begin{array}{r}3^{\circ}-08 / \\
2^{\circ}-11\end{array}$ & $\begin{array}{r}2^{\circ}-07 / \\
3^{\circ}-08\end{array}$ & $\begin{array}{r}3^{\circ}-08 / \\
2^{\circ}-11\end{array}$ \\
\hline Agricultura & -157 & -61 & $-11,4$ & $-5,0$ & -72 & -108 & $-7,8$ & $-12,7$ \\
\hline Industria & -94 & -340 & $-3,1$ & $-11,7$ & -16 & -650 & $-0,5$ & $-20,1$ \\
\hline Construcción & -88 & -136 & $-6,5$ & $-10,9$ & -350 & -933 & $-12,9$ & $-39,5$ \\
\hline Servicios tradicionales & -21 & -76 & $-0,6$ & $-2,1$ & 220 & -222 & 3,8 & $-3,7$ \\
\hline Servicios avanzados & 54 & -33 & 5,8 & $-3,4$ & 105 & -304 & 4,2 & $-11,7$ \\
\hline Servicios colectivos & 80 & 22 & 4,0 & 1,1 & 86 & 371 & 2,4 & 10,1 \\
\hline Otros servicios & 28 & -60 & 3,6 & $-7,3$ & 7 & -198 & 0,4 & $-12,1$ \\
\hline Total & -198 & -684 & $-1,5$ & $-5,3$ & -21 & -2.043 & $-0,1$ & $-10,0$ \\
\hline
\end{tabular}

Nota: "servicios tradicionales" incluye comercio, reparaciones, hostelería, transportes y comunicaciones; "servicios avanzados" incluye intermediación financiera, actividades inmobiliarias y de alquiler y servicios a empresas; "servicios colectivos" incluye administración pública, educación, actividades sanitarias y organismos extraterritoriales; "otros servicios" incluye otras actividades sociales y hogares que emplean personal doméstico.

Fuente: EPA, $2^{\circ}$ trimestres (INE).

Otra forma de aproximarse a la evolución desagregada del empleo es adoptar la perspectiva de las ocupaciones, que puede considerarse complementaria de la ofrecida por los sectores de actividad económica, ya que aporta información, no sobre el tipo de bienes o servicios producidos por las empresas y organizaciones en cuyos procesos de producción participa el factor trabajo, sino sobre las cualificaciones profesionales con que cuentan los trabajadores para participar en el proceso productivo. En este sentido, hay que señalar que, como es conocido, en la construcción predominan básicamente las tareas de tipo manual encauzadas a la transformación de materiales. Las ocupaciones de la Clasificación Nacional de Ocupaciones (CNO) relacionadas directamente con la construcción que incorporan dichas tareas se encuentran en diferentes grupos ocupacionales.

Por lo que respecta a los puestos manuales cualificados, la mayor parte se agrupa en torno al gran grupo 7 ("Artesanos y trabajadores cualificados de industria manufacturera, construcción y minería, excepto operadores"), del cual el grupo $\mathrm{M}$ engloba a "Trabajadores cualificados de construcción excepto opera- 
dores de maquinaria", en particular, "Encargados de obra y otros encargados de construcción" (ocupación 70), "Trabajadores cualificados en obras estructurales de construcción y asimilados" (ocupación 71) y "Trabajadores cualificados de acabado de construcciones y asimilados; pintores y otros" (ocupación 72). En la ocupación 71 se incluyen los trabajadores cualificados en oficios de la construcción, tales como albañiles, encofradores, ferrallistas, techadores, carpinteros, enlucidores y escayolistas, entre otros, mientras que en la ocupación 72 se encuentran los electricistas, fontaneros, pintores, soladores, etc. El resto de las ocupaciones de dicho grupo se refieren a puestos de la industria pesada y manufacturera.

También hay puestos cualificados relacionados con la construcción en el gran grupo 8 ("Operadores de instalaciones y maquinaria; montadores"), que incluye los grupos Q ("Operadores de instalaciones industriales de maquinaria fija; montadores y ensambladores") y R ("Conductores y operadores de maquinaria móvil"): mientras las ocupaciones del primer grupo están vinculadas a las ramas industriales, las del segundo grupo se encuentran en parte asociadas con la construcción ya que éste engloba las ocupaciones 85 ("Maquinista de locomotora; operador de maquinaria agrícola y de equipos pesados móviles; marineros") y 86 ("Conductores de vehículos para transporte urbano o por carretera"), dentro de las cuales (especialmente en la ocupación 85) se incluyen puestos directamente relacionados con la actividad constructora, como los que tienen que ver con la operación y el manejo de toda una serie de maquinaria especialmente importante en las obras públicas (grúas, bulldozers, excavadoras, niveladoras, hormigoneras, etc.).

En cuanto a los puestos manuales no cualificados, el gran grupo 9 recoge a "Trabajadores no cualificados", de los cuales los relacionados con la construcción se agrupan en la ocupación 96 ("Trabajadores no cualificados de la construcción"). Finalmente, por lo que respecta a las ocupaciones que agrupan tareas no manuales (administrativas y de índole directiva, técnica o científica), tampoco hay ninguna rúbrica referida estrictamente a la construcción.

Una vez realizadas estas acotaciones, veamos qué nos dicen los datos. En la Tabla 3 se ofrecen los resultados de ordenar las variaciones del empleo por ocupaciones para el período expansivo 1996-2007 y para el período recesivo $2007-2010^{3}$. Sólo se presentan las quince ocupaciones (de las 66 consideradas) que más crecieron en el primer período y que más disminuyeron en el segundo

\footnotetext{
${ }^{3}$ La Clasificación Nacional de Ocupaciones 1994 (CNO-1994) se ha utilizado en la EPA desde 1994 hasta 2010, siendo reemplazada por la nueva Clasificación Nacional de Ocupaciones 2011 (CNO-2011) en el primer trimestre de 2011, si bien a lo largo de los cuatro trimestres de 2010 las variables de ocupación se han codificado utilizando tanto la clasificación CNO-2011 como la anterior CNO-1994. Debido a este cambio, se ha preferido ofrecer la variación del empleo en la crisis actual hasta el $4^{\circ}-2010$ en vez de hasta el $2^{\circ}-2011$.
} 
en términos absolutos (también se da la información de sus variaciones relativas).

En primer lugar, las tres ocupaciones más directamente relacionadas con el sector de la construcción (ocupaciones 71, 72 y 96) se encuentran entre las que más crecieron durante la expansión. Entre las tres sumaron un aumento de casi 1,3 millones de ocupados (un 17\% del aumento neto total) y cada una de ellas vio duplicar (en el caso de los peones incluso más) su nivel de empleo entre 1996 y 2007 (frente a un incremento relativo de la ocupación total del 61\%). Otras ocupaciones asociadas indirectamente con la construcción (como "Técni$\cos$ y profesionales en ciencias físicas, químicas, matemáticas e ingeniería", "Técnicos de apoyo en ciencias físicas, químicas e ingeniería" e incluso "Técnicos de apoyo en operaciones financieras y comerciales") también experimentaron incrementos absolutos y relativos muy sustanciales.

Tabla 3

Ocupaciones que más aumentaron su empleo en el período de expansión $\left(1^{\circ}-1996 / 2^{\circ}\right.$ $2007)$ y que más redujeron su empleo en el período de recesión $\left(3^{\circ}-2007 / 4^{\circ}-2010\right)$, miles de personas

\begin{tabular}{|c|c|c|}
\hline OCUPACIONES QUE MÁS AUMENTARON EN 1996-2007 & $\begin{array}{l}\text { Variación } \\
\text { absoluta }\end{array}$ & $\begin{array}{l}\text { Variación } \\
\text { relativa }\end{array}$ \\
\hline 91 Empleados domésticos y otro personal limpieza interior edificios & 693 & 106,8 \\
\hline $\begin{array}{l}71 \text { Trabajadores cualificados en obras estructurales de construcción y } \\
\text { asimilados }\end{array}$ & 551 & 111,4 \\
\hline 50 Trabajadores servicios de restauración & 504 & 104,8 \\
\hline 34 Técnicos de apoyo: en gestión administrativa & 463 & 133,3 \\
\hline 51 Trabajadores en servicios personales & 401 & 98,3 \\
\hline 53 Dependientes de comercio y asimilados & 398 & 64,0 \\
\hline $\begin{array}{l}72 \text { Trabajadores cualificados de acabado de construcciones y asimilados; } \\
\text { pintores y otros }\end{array}$ & 382 & 99,6 \\
\hline 33 Técnicos de apoyo: en operaciones financieras y comerciales & 371 & 123,6 \\
\hline 30 Técnicos de apoyo: en ciencias físicas, químicas e ingeniería & 323 & 158,4 \\
\hline 96 Peones de la construcción & 279 & 132,5 \\
\hline $\begin{array}{l}44 \text { Auxiliares administrativos (con tareas de atención al público) no } \\
\text { clasificados anterior. }\end{array}$ & 239 & 77,3 \\
\hline 11 Dirección de empresas & 230 & 100,4 \\
\hline 22 Técnicos y profesionales: en enseñanza & 223 & 84,4 \\
\hline $\begin{array}{l}20 \text { Técnicos y profesionales: en ciencias físicas, químicas, matemáticas e } \\
\text { ingeniería }\end{array}$ & 199 & 198,0 \\
\hline 86 Conductores de vehículos para transporte urbano o por carretera & 197 & 34,1 \\
\hline Total & 7.741 & 61,3 \\
\hline
\end{tabular}


Tabla 3 (continuación)

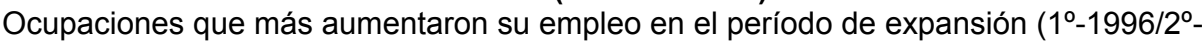
2007) y que más redujeron su empleo en el período de recesión $\left(3^{\circ}-2007 / 4^{\circ}-2010\right)$, miles de personas

\begin{tabular}{|c|c|c|}
\hline OCUPACIONES QUE MÁS DISMINUYERON EN 2007-2010 & $\begin{array}{l}\text { Variación } \\
\text { absoluta }\end{array}$ & $\begin{array}{l}\text { Variación } \\
\text { relativa }\end{array}$ \\
\hline $\begin{array}{l}71 \text { Trabajadores cualificados en obras estructurales de construcción y } \\
\text { asimilados }\end{array}$ & -490 & $-46,8$ \\
\hline 96 Peones de la construcción & -292 & $-64,3$ \\
\hline $\begin{array}{l}72 \text { Trabajadores cualificados de acabado de construcciones y asimilados; } \\
\text { pintores y otros }\end{array}$ & -226 & $-28,8$ \\
\hline 50 Trabajadores en servicios de restauración & -192 & $-18,4$ \\
\hline 91 Empleados domésticos y otro personal limpieza interior edificios & -127 & $-9,1$ \\
\hline $\begin{array}{l}44 \text { Auxiliares administrativos (con tareas de atención al público) no } \\
\text { clasificados anterior. }\end{array}$ & -107 & $-18,4$ \\
\hline $\begin{array}{l}75 \text { Soldadores, chapistas, montadores de estructuras metálicas, herreros, } \\
\text { elaboración de herramientas y asimilados }\end{array}$ & -106 & $-32,1$ \\
\hline $\begin{array}{l}76 \text { Mecánicos y ajustadores de maquinaria y equipos eléctricos y } \\
\text { electrónicos }\end{array}$ & -82 & $-14,8$ \\
\hline 53 Dependientes de comercio y asimilados & -76 & $-7,3$ \\
\hline $\begin{array}{l}79 \text { Trabajadores cualificados del tratamiento de madera, ebanistas, } \\
\text { industria textil, confección, piel, cuero, calzado y asimilados }\end{array}$ & -73 & $-42,2$ \\
\hline 83 Operadores de máquinas fijas & -68 & $-15,5$ \\
\hline 33 Técnicos y profesionales: en operaciones financieras y comerciales & -68 & $-10,0$ \\
\hline 86 Conductores de vehículos para transporte urbano o por carretera & -66 & $-8,6$ \\
\hline $\begin{array}{l}85 \text { Maquinista de locomotora; operador de maquinaria agrícola y de } \\
\text { equipos pesados móviles; marineros }\end{array}$ & -64 & $-23,0$ \\
\hline 70 Encargados de obra y otros encargados de construcción & -52 & $-43,9$ \\
\hline Total & -2.102 & $-10,3$ \\
\hline
\end{tabular}

Fuente: EPA (INE).

En segundo lugar, las tres ocupaciones mencionadas anteriormente (ocupaciones 71,72 y 96) han sido las que más se han reducido durante la recesión. Si se añaden las ocupaciones 70 y 85 , entre las cinco suman una disminución neta del empleo de más de 1,1 millones, es decir, algo más de la mitad de la pérdida total de ocupación hasta finales de 2010. Las ocupaciones 70 y 71 pierden casi la mitad de los efectivos que tenían en 2007, mientras que la 96 pierde casi dos tercios y la 72 y 85 en torno a una cuarta parte, lo que contrasta con la reducción del 10,3\% del empleo total.

Estos resultados obtenidos a partir de la información relacionada con las ocupaciones asociadas directa o indirectamente con la construcción abundan en el análisis realizado previamente con los datos sectoriales. 


\section{EL CASO ESPAÑOL EN EL CONTEXTO EUROPEO}

Una vez examinada la evolución de la ocupación en general y la del sector de la construcción en particular en España, podemos preguntarnos si ésta ha sido diferente en comparación con el resto de países de la Unión Europea. Para ayudar a contestar esta pregunta, la Tabla 4 ofrece la información sobre el empleo total, el empleo no agrario y el empleo en la construcción en los años 1999, 2007 y 2010 procedente de la Encuesta de Fuerza de Trabajo de la Unión Europea de Eurostat. Los datos se presentan en forma de índice, de modo que los niveles de empleo en el año 1999 toman el valor 100 (excepto para los agregados UE-25 y UE-27, que se refieren al año 2000). Este cuadro también proporciona la evolución del peso de la ocupación en la construcción en relación con la ocupación total y con la ocupación no agraria en dichos años. Los países se encuentran ordenados según el nivel de empleo en la construcción alcanzado en 2007 en relación con su nivel en 1999.

Como se desprende de los datos de la Tabla, España superó con creces la media europea de aumento del empleo total y del empleo no agrario: fue el país que creó más puestos de trabajo, de modo que su nivel de ocupación total en 2007 era casi un $40 \%$ más elevado que en 1999 , frente a una media europea cercana al 10\% (UE-25 y UE-27). Sólo Chipre e Irlanda muestran unos resultados que se acercan a los españoles en cuanto a empleo total. Pero además España fue uno de los países que aumentó más su ocupación en la construcción, sólo superado por los tres países bálticos e Irlanda y con cifras similares a Rumanía: mientras en Estonia y Letonia el empleo en la construcción se multiplicó casi por 2,2 y en Irlanda por 2, en Lituania, España y Rumanía, se multiplicó por algo más de 1,7 .

Esta evolución de la ocupación total y en la construcción llevó a que el peso de este sector en el empleo total aumentase en más de 5 puntos en los dos primeros países, en torno a 4,5 puntos en Irlanda y Lituania, 3,5 puntos en $\mathrm{Ru}$ manía y 2,5 puntos en España (la media europea se situó en torno a 0,5 puntos). Como resultado de este proceso, España, que partía de un peso de la construcción en el empleo total $(10,7 \%)$ que era el más elevado junto a Portugal en 1999, con notables diferencias con respecto a los países mencionados e incluso con respecto a la media europea (por debajo del 8\%), se mantuvo en dicha posición junto a Irlanda en 2007 (con un peso del 13,3\%), muy lejos de la media europea (que sólo subió unas décimas para situarse por encima del 8\%) y seguida por los países mencionados (excepto Rumanía), que se situaron en cabeza de los países europeos con pesos de la construcción por encima del 11\%. 


\section{Tabla 4}

Evolución del empleo total y del empleo de la construcción (índice 1999=100) y del peso de la construcción respecto al empleo total y al empleo no agrario en los países de la

Unión Europea

\begin{tabular}{|c|c|c|c|c|c|c|c|c|c|c|c|c|c|c|c|c|}
\hline & \multicolumn{6}{|c|}{ EVOLUCIÓN EMPLEO $(199=100)$} & \multicolumn{5}{|c|}{$\begin{array}{c}\text { PESO } \\
\text { CONSTRUCCIÓN/EMPLEO } \\
\text { TOTAL } \\
\end{array}$} & \multicolumn{5}{|c|}{$\begin{array}{c}\text { PESO } \\
\begin{array}{c}\text { CONSTRUCCIÓN/EMPLEO NO } \\
\text { AGRARIO }\end{array} \\
\end{array}$} \\
\hline & \multicolumn{3}{|c|}{2007} & \multicolumn{3}{|c|}{2010} & \multicolumn{3}{|c|}{ NIVEL } & \multicolumn{2}{|c|}{ VARIACIÓN } & \multicolumn{3}{|c|}{ NIVEL } & \multicolumn{2}{|c|}{ VARIACIÓN } \\
\hline & $\left|\begin{array}{c}\text { Empleo } \\
\text { total }\end{array}\right|$ & \begin{tabular}{|c|} 
Empleo \\
no \\
agrario
\end{tabular} & Constr. & $\begin{array}{c}\text { Empleo } \\
\text { total }\end{array}$ & \begin{tabular}{|c|}
$\begin{array}{c}\text { Empleo } \\
\text { no } \\
\text { agrario }\end{array}$ \\
\end{tabular} & Constr. & 1999 & |2007| & 2010 & $\begin{array}{l}1999- \\
2007\end{array}$ & $\begin{array}{l}2007- \\
2010\end{array}$ & 1999 & 2007 & 2010 & $\begin{array}{c}1999- \\
2007\end{array}$ & $\begin{array}{l}2007- \\
2010\end{array}$ \\
\hline Estonia & 113,0 & 117,8 & 217,5 & 98,4 & 103,1 & 128,8 & 6,4 & 12,3 & 8,4 & 5,9 & $-4,0$ & 7,0 & 13,0 & 8,8 & 5,9 & $-4,2$ \\
\hline Letonia & 115,0 & 125,6 & 214,8 & 96,8 & 107,1 & 112,7 & 6,1 & 11,3 & 7,1 & 5,3 & $-4,3$ & 7,3 & 12,6 & 7,7 & 5,2 & $-4,8$ \\
\hline Irlanda & 132,9 & 137,4 & 197,7 & 116,0 & 121,2 & 83,4 & 8,9 & 13,3 & 6,4 & 4,4 & $-6,9$ & 9,8 & 14,1 & 6,7 & 4,3 & $-7,3$ \\
\hline Lituania & 103,1 & 115,5 & 175,1 & 90,3 & 102,7 & 95,6 & 6,6 & 11,1 & 6,9 & 4,6 & $-4,2$ & 8,2 & 12,4 & 7,6 & 4,2 & $-4,8$ \\
\hline España & 138,6 & 142,4 & 171,6 & 125,6 & 129,5 & 105,0 & 10,7 & 13,3 & 8,9 & 2,5 & $-4,3$ & 11,5 & 13,9 & 9,3 & 2,4 & $-4,5$ \\
\hline Rumanía & 87,8 & 107,2 & 170,9 & 86,8 & 105,0 & 177,5 & 3,7 & 7,3 & 7,6 & 3,5 & 0,4 & 6,5 & 10,3 & 10,9 & 3,8 & 0,6 \\
\hline Chipre & 135,3 & 135,7 & 162,5 & 137,9 & 139,1 & 158,2 & 9,8 & 11,8 & 11,3 & 2,0 & $-0,5$ & 10,3 & 12,4 & 11,7 & 2,0 & $-0,6$ \\
\hline Grecia & 111,9 & 120,6 & 138,4 & 108,9 & 116,0 & 112,9 & 7,1 & 8,7 & 7,3 & 1,7 & $-1,4$ & 8,6 & 9,9 & 8,4 & 1,3 & $-1,5$ \\
\hline Suecia & 112,0 & 112,9 & 130,5 & 112,1 & 113,2 & 137,6 & 5,5 & 6,4 & 6,7 & 0,9 & 0,3 & 5,6 & 6,5 & 6,8 & 0,9 & 0,3 \\
\hline Hungría & 103,6 & 106,1 & 129,0 & 99,7 & 102,3 & 108,3 & 6,8 & 8,4 & 7,3 & 1,7 & $-1,1$ & 7,3 & 8,8 & 7,7 & 1,6 & $-1,1$ \\
\hline Eslovenia & 111,6 & 112,1 & 125,6 & 109,4 & 111,2 & 123,9 & 5,3 & 6,0 & 6,0 & 0,7 & 0,0 & 5,9 & 6,6 & 6,6 & 0,7 & 0,0 \\
\hline Eslovaquia & 110,6 & 114,4 & 125,1 & 108,7 & 113,6 & 136,2 & 8,9 & 10,1 & 11,1 & 1,2 & 1,1 & 9,6 & 10,5 & 11,5 & 0,9 & 1,0 \\
\hline Reino Unido & 106,4 & 106,6 & 124,7 & 108,3 & 108,6 & 119,0 & 7,0 & 8,2 & 7,6 & 1,2 & $-0,5$ & 7,1 & 8,3 & 7,7 & 1,2 & $-0,5$ \\
\hline Italia & 112,2 & 114,0 & 124,1 & 110,5 & 112,5 & 123,7 & 7,6 & 8,4 & 8,5 & 0,8 & 0,1 & 8,1 & 8,8 & 8,9 & 0,7 & 0,1 \\
\hline Francia & 116,3 & 117,4 & 121,8 & 114,3 & 115,9 & 131,6 & 6,4 & 6,7 & 7,4 & 0,3 & 0,7 & 6,7 & 7,0 & 7,6 & 0,3 & 0,7 \\
\hline UE 27 & 108,5 & 111,0 & 116,9 & 107,2 & 110,2 & 108,5 & 7,6 & 8,2 & 7,7 & 0,6 & $-0,5$ & 8,2 & 8,6 & 8,1 & 0,4 & $-0,6$ \\
\hline Finlandia & 108,5 & 110,5 & 116,6 & 106,6 & 108,7 & 115,5 & 6,5 & 7,0 & 7,0 & 0,5 & 0,1 & 6,9 & 7,3 & 7,4 & 0,4 & 0,1 \\
\hline Euro Zona 12 & 113,9 & 115,4 & 116,0 & 114,9 & 116,8 & 107,6 & 8,1 & 8,3 & 7,6 & 0,1 & $-0,7$ & 8,6 & 8,6 & 7,9 & 0,0 & $-0,7$ \\
\hline UE 25 & 109,5 & 111,0 & 114,7 & 108,3 & 110,3 & 106,1 & 7,8 & 8,2 & 7,6 & 0,4 & $-0,5$ & 8,3 & 8,6 & 8,0 & 0,3 & $-0,6$ \\
\hline Bélgica & 109,3 & 109,5 & 110,8 & 112,0 & 112,7 & 118,3 & 6,8 & 6,9 & 7,2 & 0,1 & 0,3 & 6,9 & 7,0 & 7,3 & 0,1 & 0,3 \\
\hline Países Bajos & 111,3 & 111,4 & 109,5 & 110,1 & 110,4 & 98,8 & 6,1 & 6,0 & 5,4 & $-0,1$ & $-0,5$ & 6,3 & 6,2 & 5,6 & $-0,1$ & $-0,6$ \\
\hline Luxemburgo & 115,2 & 115,3 & 108,3 & 125,4 & 126,5 & 89,2 & 8,9 & 8,4 & 6,3 & $-0,5$ & $-2,0$ & 9,1 & 8,5 & 6,4 & $-0,6$ & $-2,1$ \\
\hline Portugal & 105,3 & 106,5 & 106,2 & 101,4 & 103,4 & 89,8 & 10,9 & 11,0 & 9,7 & 0,1 & $-1,4$ & 12,5 & 12,5 & 10,9 & 0,0 & $-1,6$ \\
\hline Dinamarca & 103,9 & 104,1 & 106,0 & 100,7 & 101,5 & 87,2 & 6,8 & 7,0 & 5,9 & 0,1 & $-1,1$ & 7,0 & 7,2 & 6,0 & 0,1 & $-1,1$ \\
\hline Austria & 109,1 & 109,6 & 101,5 & 110,9 & 112,1 & 105,1 & 8,8 & 8,2 & 8,3 & $-0,6$ & 0,1 & 9,4 & 8,7 & 8,8 & $-0,7$ & 0,1 \\
\hline Rep. Checa & 104,4 & 106,2 & 100,8 & 103,6 & 106,0 & 104,9 & 9,4 & 9,1 & 9,5 & $-0,3$ & 0,4 & 9,9 & 9,4 & 9,8 & $-0,5$ & 0,4 \\
\hline Alemania & 105,9 & 106,5 & 80,3 & 107,3 & 108,7 & 82,1 & 8,7 & 6,6 & 6,7 & $-2,1$ & 0,1 & 9,0 & 6,8 & 6,8 & $-2,2$ & 0,0 \\
\hline
\end{tabular}

Notas:

- Los niveles de empleo total y de cada sector en cada país toman el valor 100 en el año 1999 (excepto para UE27 y UE-25 que se refieren al año 2000).

- No aparecen Bulgaria, Malta y Polonia por carecer de información en dicho año. Por ello, UE-27 no incluye a estos países.

Fuente: Encuesta de Fuerza de Trabajo de la UE (Eurostat). 
El período recesivo ha implicado una pérdida de puestos de trabajo que ha llevado a algunos países a tener un nivel de empleo total en 2010 inferior al de 1999 (precisamente los países que acabamos de mencionar, menos Irlanda), si bien eso no ha ocurrido si se considera el empleo no agrario. España, a pesar de la enorme destrucción de puestos, era el país que en 2010 tenía un nivel de ocupación más alta en comparación con 1999 (con excepción de Chipre). Sin embargo, el nivel de empleo en la construcción retrocedió en el caso español casi a los niveles de dicho año, lo que contrasta con la mayoría de los países europeos (en casi todos ellos la ocupación en la construcción cae algo pero también hay casos en que aumenta) aunque no con los cinco países (excepto Rumanía) que hemos citado antes, en los que las pérdidas en la construcción fueron enormes: en Estonia y Letonia el empleo en la construcción se mantuvo por encima de su nivel en 1999 pero en Lituania y, sobre todo, Irlanda cayó por debajo de dicho nivel.

El resultado de esta evolución de la ocupación total y en la construcción fue una reducción del peso de este sector en el empleo total de algo más de 4 puntos en Estonia, Letonia, Lituania y España y casi 7 puntos en Irlanda. De hecho, España e Irlanda han sido los únicos países en que la disminución del peso de la ocupación de la construcción en la ocupación total en el período 2007-2010 ha superado el aumento de dicho peso en el período 1999-2007. En consecuencia, ambos países muestran en 2010 pesos de la construcción inferiores a los de 1999, si bien España continúa teniendo uno que se encuentra por encima de la media y entre los más elevados de los países europeos.

Para finalizar esta comparación entre los países europeos, las Figuras 2 y 3 ofrecen las correlaciones entre la variación del peso del empleo en la construcción con respecto al empleo total y la variación de la tasa de paro, por un lado, y entre la variación de dicho peso y la variación de la tasa de ocupación, por otro lado. Las 48 observaciones que componen la nube de puntos y que aparecen en cada una de las Figuras representan la relación entre ambas variables para cada uno de los países en los dos períodos considerados más arriba (1999-2007 y 2007-2010), lo que significa que cada país aparece dos veces en cada gráfico. Todas las variables están medidas en puntos porcentuales. Los datos en los que se basan ambos gráficos se encuentran en la Tabla A.1 del Apéndice.

Los resultados abundan en los hallazgos comentados más arriba, puesto que ponen de manifiesto la relación claramente negativa (positiva) entre las variaciones experimentadas por la participación del sector de la construcción en el empleo total y las variaciones de la tasa de paro (tasa de ocupación) tanto en la etapa expansiva como en la recesiva. La mayor parte de los puntos se encuentran en el segundo y el cuarto cuadrantes, en un caso, y en el primero y el tercero, en otro caso, y los coeficientes de las rectas de regresión ajustadas indican que una reducción de un punto porcentual en el peso de la construcción se en- 
cuentra asociada con una elevación (disminución) de la tasa de paro (de la tasa de ocupación) de 1,7 $(1,4)$ puntos porcentuales, en media.

\section{Figura 2}

Variación del peso del empleo en la construcción con respecto al empleo total y variación de la tasa de paro (en puntos porcentuales), 1999-2007 y 2007-2010

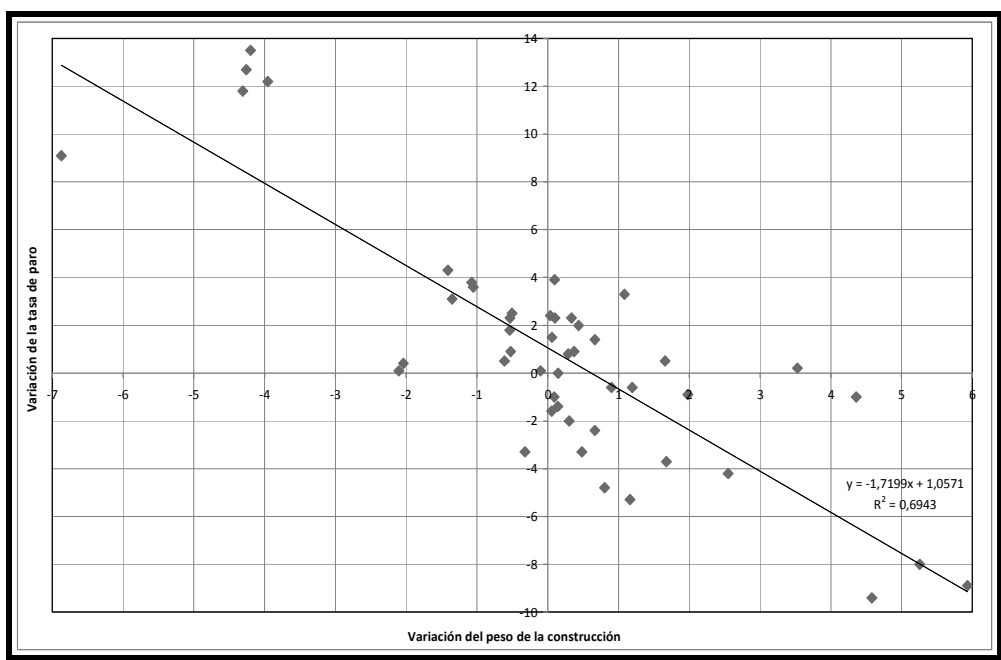

Fuente: Encuesta de Fuerza de Trabajo de la UE (Eurostat).

Figura 3

Variación del peso del empleo en la construcción con respecto al empleo total y variación de la tasa de ocupación (en puntos porcentuales), 1999-2007 y 2007-2010

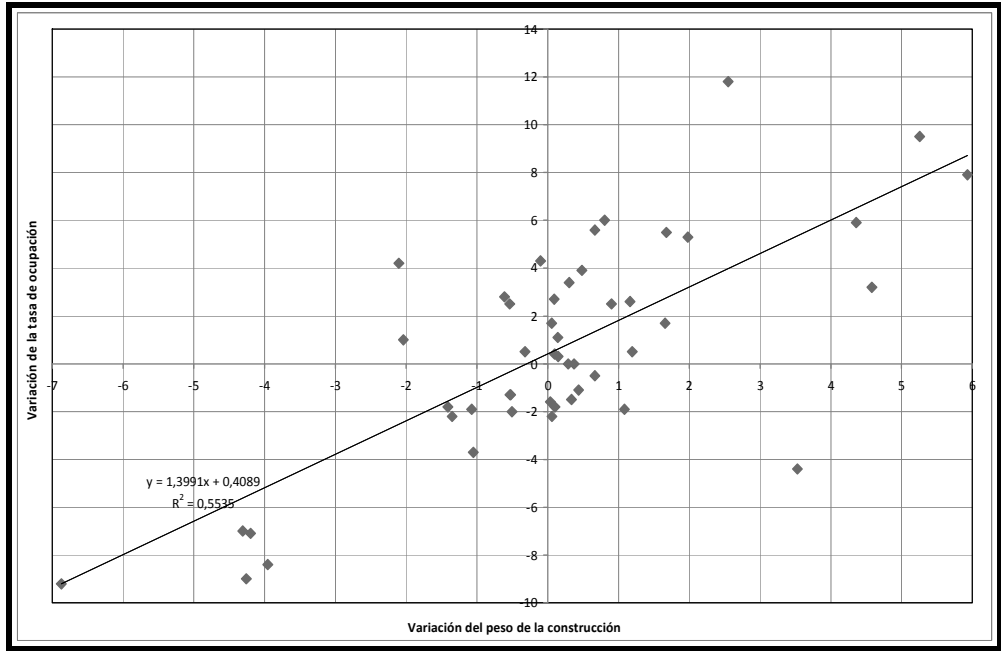

Fuente: Encuesta de Fuerza de Trabajo de la UE (Eurostat). 


\section{CONCLUSIONES}

En este artículo se ha pretendido ofrecer una panorámica de la evolución de la ocupación en la construcción en España en el contexto tanto del empleo total de la economía y del resto de los sectores productivos en los últimos veinticinco años como de la ocupación en los países de nuestro entorno en la última década.

La comparación temporal ha llevado a concluir que el sector de la construcción posee características claramente cíclicas, lo que lleva a que su ocupación aumente más que el resto de los sectores en épocas expansivas y disminuya más en épocas recesivas, de modo que su peso en el empleo está sujeto a estas variaciones, elevándose en las expansiones y reduciéndose en las recesiones. La última expansión, sin embargo, fue más duradera que la anterior, lo que llevó al nivel de empleo y al peso de la construcción a superar con creces el nivel más alto alcanzado en 1991. Además, a diferencia de lo ocurrido en la crisis anterior (1991-1994), la pérdida de empleo comenzó en el sector de la construcción en la primera fase de la recesión y posteriormente se intensificó en dicho sector y se extendió al resto de los sectores, especialmente a la industria pero también a los servicios, en particular las actividades financieras, actividades inmobiliarias y servicios a las empresas.

La comparación con lo sucedido en los países del entorno europeo sugiere que el período expansivo fue mucho más intensivo en creación de empleo en España que en ningún otro país y que el aumento de la ocupación total estuvo muy relacionado con el incremento de la ocupación en la construcción: sólo los tres países bálticos e Irlanda superaron a España en aumento neto de empleo en este sector, elevando su peso en el empleo total 4,5-5,5 puntos en dichos países y 2,5 puntos en España (frente a una media europea de alrededor de 0,5 puntos). La recesión trajo consigo una enorme pérdida de puestos de trabajo en la construcción y una gran reducción del peso del sector en el empleo total en estos países, elevando sus tasas de paro en términos relativos en cifras similares en todos ellos (nueve puntos porcentuales o más) y reduciendo sus tasas de ocupación de manera comparable (siete puntos porcentuales o más), lo que da una idea del impacto del pinchazo del sector inmobiliario sobre las cifras agregadas de empleo y paro. España e Irlanda han sido los únicos países en que la disminución del peso de la ocupación de la construcción en la ocupación total en el período recesivo ha superado el aumento de dicho peso en el período expansivo.

En suma, ambas perspectivas (la temporal y la internacional) sugieren que la grave crisis de la economía y del empleo en España (de la misma forma que en otros países), cualesquiera que sean sus causas últimas, está muy ligada al hundimiento del sector de la construcción que se inició en la segunda mitad del año 2007 y se extendió a lo largo de los años 2008-2011, que arrastró a otros sectores tanto por la vía de la demanda específica de bienes, en el caso de los sectores vinculados a la construcción, como por la vía del descenso general de 
la demanda agregada que acompaña a cualquier caída de los ingresos como la que trajo consigo la fuerte destrucción de empleo. Por tanto, sin negar que puede haber otros factores relacionados con el modelo productivo español y con el funcionamiento del mercado de trabajo que generan y acentúan las fluctuaciones tan enormes del empleo y del paro en España en comparación con la mayor parte de los países europeos (Costain et al., 2009; Sala y Silva, 2009; Pérez Infante, 2010; García-Serrano, 2011), parece que la construcción estuvo en el arranque de la recesión española y su crisis acabó arrastrando al resto de la economía al tiempo que España sufría un intenso shock negativo adicional por los problemas del sistema financiero internacional. Esto último ha repercutido en generar una escasez de financiación de las empresas que extiende sus efectos negativos a todos los sectores.

\section{REFERENCIAS BIBLIOGRÁFICAS}

COSTAIN, J.; JIMENO, J.F. y THOMAS, C. (2009). "El funcionamiento del mercado de trabajo y el aumento del paro en España" en Boletín Económico del Banco de España, julio-agosto, pp. 97-115.

ENGLUND, P. y IOANNIDES, Y.M. (1997). "House price dynamics: an international empirical perspective" en Journal of Housing Economics, 6, pp. 119136.

FELGUEROSO, F. (2011). "El mercado de trabajo en la Gran Recesión (I): 'el pánico a contratar' y el 'pánico a moverse'” en Nada es Gratis, junio. http://www.fedeablogs.net/economia/?p=12625. [Último acceso: 12 de noviembre de 2011].

FONDO MONETARIO INTERNACIONAL (2004). "Tres cuestiones actuales de política económica" en World Economic Outlook, septiembre.

GARCÍA-MONTALVO, J. (2006). "Deconstruyendo la burbuja: expectativas de revalorización y precio de la vivienda en España" en Papeles de Economía Española, 109, pp. 42-75.

GARCÍA-MONTALVO, J. (2009). "Financiación inmobiliaria, burbuja crediticia y crisis financiera: lecciones a partir de la recesión de 2008-09" en Papeles de Economía Española, 122, pp. 66-87.

GARCÍA-SERRANO, C. (2011). "Déjà vu? Crisis de empleo y reformas laborales en España" en Revista de Economía Española, 56(XIX), pp. 149-177.

KASPAROVA, D. y WHITE, M. (2001). "The responsiveness of house prices to macroeconomic forces: a cross-country comparison" en European Journal of Housing Policy, 1, pp. 385-416. 
KIM, K.H. (1999). "Korea: could a real estate price bubble have caused the economic crisis?" en Documento de Trabajo, Sogang University.

OCDE (2005). "Recent house developments: the role of fundamentals" en Economic Outlook, 78 (diciembre).

MALPEZZI, S. y WACHTER, S. (2005). "The role of speculation in real estate cycles" en Journal of Real Estate Literature, 13, pp. 143-166.

MEEN, G. (1990). "The removal of mortgage market constraints and the implications for econometric modelling of UK house prices" en Oxford Bulletin of Economics and Statistics, 52, pp. 1-23.

MEEN, G. (1996). "Ten propositions in UK housing macroeconomics: an overview of the 1980s and 1990s" en Urban Studies, 33, pp. 425-444.

PÉREZ INFANTE, J.I. (2010). "El mercado de trabajo español y la última reforma laboral" en Boletín de Inflación y Análisis Macroeconómico, Instituto Flores de Lemus de la Universidad Carlos III de Madrid, 190, julio, pp. 69-77.

RODRÍGUEZ, J. (2006). "Los booms inmobiliarios en España. Un análisis de tres períodos" en Papeles de Economía Española, 109, pp. 76-90.

SALA, H. y SILVA, J.I. (2009). "Flexibility at the margin and labour market volatility: the case of Spain" en Investigaciones Económicas, 33(2), pp. 1145178.

STEVENSON, S. y YOUNG, J. (2006). "Comportamiento especulativo en los mercados de la vivienda: una perspectiva internacional" en Papeles de Economía Española, 109, pp. 14-26.

THE ECONOMIST (2005). "Income the waves: the global housing boom", 16 de junio.

TOHARIA, L. (1996a). "Empleo y paro en España: evolución, situación y perspectivas" en Ekonomiaz, 35, pp. 35-67.

TOHARIA, L. (1996b). "La medición del empleo y el paro en España" en Cuadernos de Información Económica, 108, pp. 20-28.

TOHARIA, L.; GARCÍA-SERRANO, C. y SAHÚN, M.P. (1991). El factor trabajo en la construcción: empleo, demanda y oferta (1985-1990), y la formación profesional en el sector. Madrid: Secretaría General Técnica, Centro de Publicaciones, Ministerio de Obras Públicas y Transportes.

TOHARIA, L. y MALO, M.A. (2009). "¿Qué se puede esperar de las reformas del mercado de trabajo?" en V. Gómez (ed.): La reforma laboral en España (pp. 125-156). Madrid: Fundación Ortega y Gasset.

WHITEHEAD, C. (2006). "Una perspectiva internacional de los mercados de la vivienda" en Papeles de Economía Española, 109, pp. 2-13. 


\section{Apéndice}

Tabla A.1.

Variación del peso de la construcción respecto al empleo total y de las tasas de paro y ocupación en los países de la Unión Europea (puntos porcentuales)

\begin{tabular}{|c|c|c|c|c|c|c|c|c|c|c|c|c|}
\hline & \multicolumn{3}{|c|}{$1999-2007$} & \multicolumn{3}{|c|}{ 2007-2010 } & \multicolumn{3}{|c|}{ Tasa de paro } & \multicolumn{3}{|c|}{$\begin{array}{c}\text { Tasa de ocupación } \\
\text { (15-64 años) }\end{array}$} \\
\hline & $\begin{array}{c}\text { Variac. } \\
\text { peso } \\
\text { constr. }\end{array}$ & $\begin{array}{c}\text { Variac. } \\
\text { tasa } \\
\text { paro }\end{array}$ & $\begin{array}{c}\text { Variac. } \\
\text { tasa } \\
\text { ocup. }\end{array}$ & $\begin{array}{c}\text { Variac. } \\
\text { peso } \\
\text { constr. }\end{array}$ & $\begin{array}{c}\text { Variac. } \\
\text { tasa } \\
\text { paro }\end{array}$ & $\begin{array}{c}\text { Variac. } \\
\text { tasa } \\
\text { ocup. }\end{array}$ & 1999 & 2007 & 2010 & 1999 & 2007 & 2010 \\
\hline UE 27 & 0,6 & 1,6 & 3,6 & $-0,5$ & 2,5 & $-1,3$ & 8,8 & 7,2 & 9,7 & 51,8 & 65,4 & 64,1 \\
\hline UE 25 & 0,4 & $-1,9$ & 3,9 & $-0,5$ & 2,5 & $-1,3$ & 9,2 & 7,3 & 9,8 & 61,9 & 65,8 & 64,5 \\
\hline Euro Zona 12 & 0,1 & $-1,9$ & 4,4 & $-0,7$ & 2,5 & $-1,5$ & 9,5 & 7,6 & 10,1 & 62,5 & 66,9 & 65,4 \\
\hline Bélgica & 0,1 & $-1,0$ & 2,7 & 0,3 & 0,8 & 0,0 & 8,5 & 7,5 & 8,3 & 59,3 & 62,0 & 62,0 \\
\hline República Checa & $-0,3$ & $-3,3$ & 0,5 & 0,4 & 2,0 & $-1,1$ & 8,6 & 5,3 & 7,3 & 65,6 & 66,1 & 65,0 \\
\hline Dinamarca & 0,1 & $-1,4$ & 1,1 & $-1,1$ & 3,6 & $-3,7$ & 5,2 & 3,8 & 7,4 & 76,0 & 77,1 & 73,4 \\
\hline Alemania & $-2,1$ & 0,1 & 4,2 & 0,1 & $-1,6$ & 1,7 & 8,6 & 8,7 & 7,1 & 65,2 & 69,4 & 71,1 \\
\hline Estonia & 5,9 & $-8,9$ & 7,9 & $-4,0$ & 12,2 & $-8,4$ & 13,6 & 4,7 & 16,9 & 61,5 & 69,4 & 61,0 \\
\hline Irlanda & 4,4 & $-1,0$ & 5,9 & $-6,9$ & 9,1 & $-9,2$ & 5,6 & 4,6 & 13,7 & 63,3 & 69,2 & 60,0 \\
\hline Grecia & 1,7 & $-3,7$ & 5,5 & $-1,4$ & 4,3 & $-1,8$ & 12,0 & 8,3 & 12,6 & 55,9 & 61,4 & 59,6 \\
\hline España & 2,5 & $-4,2$ & 11,8 & $-4,3$ & 11,8 & $-7,0$ & 12,5 & 8,3 & 20,1 & 53,8 & 65,6 & 58,6 \\
\hline Francia & 0,3 & $-2,0$ & 3,4 & 0,7 & 1,4 & $-0,5$ & 10,4 & 8,4 & 9,8 & 60,9 & 64,3 & 63,8 \\
\hline Italia & 0,8 & $-4,8$ & 6,0 & 0,1 & 2,3 & $-1,8$ & 10,9 & 6,1 & 8,4 & 52,7 & 58,7 & 56,9 \\
\hline Chipre & 2,0 & $-0,9$ & 5,3 & $-0,5$ & 2,3 & $-1,3$ & 4,8 & 3,9 & 6,2 & 65,7 & 71,0 & 69,7 \\
\hline Letonia & 5,3 & $-8,0$ & 9,5 & $-4,3$ & 12,7 & $-9,0$ & 14,0 & 6,0 & 18,7 & 58,8 & 68,3 & 59,3 \\
\hline Lituania & 4,6 & $-9,4$ & 3,2 & $-4,2$ & 13,5 & $-7,1$ & 13,7 & 4,3 & 17,8 & 61,7 & 64,9 & 57,8 \\
\hline Luxemburgo & $-0,5$ & 1,8 & 2,5 & $-2,0$ & 0,4 & 1,0 & 2,4 & 4,2 & 4,6 & 61,7 & 64,2 & 65,2 \\
\hline Hungría & 1,7 & , & 1,7 & $-1,1$ & 3,8 & $-1,9$ & 6,9 & 7,4 & 11,2 & 55,6 & 57,3 & 55,4 \\
\hline Países Bajos & $-0,1$ & 0,1 & 4,3 & $-0,5$ & 0,9 & $-1,3$ & 3,5 & 3,6 & 4,5 & 71,7 & 76,0 & 74,7 \\
\hline Austria & $-0,6$ & 0,5 & 2,8 & 0,1 & 0,0 & 0,3 & 3,9 & 4,4 & 4,4 & 68,6 & 71,4 & 71,7 \\
\hline Portugal & 0,1 & 3,9 & 0,4 & $-1,4$ & 3,1 & $-2,2$ & 5,0 & 8,9 & 12,0 & 67,4 & 67,8 & 65,6 \\
\hline Rumanía & 3,5 & 0,2 & $-4,4$ & 0,4 & 0,9 & 0,0 & 6,2 & 6,4 & 7,3 & 63,2 & 58,8 & 58,8 \\
\hline Eslovenia & 0,7 & $-2,4$ & 5,6 & 0,0 & 2,4 & $-1,6$ & 7,3 & 4,9 & 7,3 & 62,2 & 67,8 & 66,2 \\
\hline Eslovaquia & 1,2 & $-5,3$ & 2,6 & 1,1 & 3,3 & $-1,9$ & 16,4 & 11,1 & 14,4 & 58,1 & 60,7 & 58,8 \\
\hline Finlandia & 0,5 & $-3,3$ & 3,9 & 0,1 & 1,5 & $-2,2$ & 10,2 & 6,9 & 8,4 & 66,4 & 70,3 & 68,1 \\
\hline Suecia & 0,9 & $-0,6$ & 2,5 & 0,3 & 2,3 & $-1,5$ & 6,7 & 6,1 & 8,4 & 71,7 & 74,2 & 72,7 \\
\hline Reino Unido & 1,2 & $-0,6$ & 0,5 & $-0,5$ & 2,5 & $-2,0$ & 5,9 & 5,3 & 7,8 & 71,0 & 71,5 & 69,5 \\
\hline
\end{tabular}

Notas:

- Las tasas de paro de UE-27, Estonia y Chipre se refieren al año 2000, no a 1999.

- La tasa de ocupación de Chipre se refiere al año 2000, no a 1999.

Fuente: Encuesta de Fuerza de Trabajo de la UE (Eurostat). 